\title{
Role of endothelin receptor A and NADPH oxidase in vascular abnormalities
}

\author{
This article was published in the following Dove Press journal: \\ Vascular Health and Risk Management \\ 28 August 2010 \\ Number of times this article has been viewed
}

\section{De-Zai Dai \\ Yin Dai}

Research Division of Pharmacology, China Pharmaceutical University, Nanjing, 210009, China
Correspondence: De-Zai Dai

076 Post Box, Chian Pharmaceutical University, 24 Tongjia Lane, Nanjing, 210009, China

Tel +86 $258327 \quad 1270$

$\mathrm{Fax}+862583302827$

Email dezaidai@vip.sina.com
Abstract: Vascular dilatation is critically impaired in many diseases and is encountered by an upregulated endothelin receptor A (ETA) in the vasculature in association with a decline in nitric oxide bioavailability. Diabetic vasculopathy is characterized as a compromised vascular dilatation, implicated in many diabetic complications. It appears to be activated ETA and NADPH (nicotinamide adenine dinucleotide phosphate) oxidase in the vasculature. Glucose-lowering agents do not always blunt these changes, as these changes may be progressive leading to the end stage of renal disease. The vascular insults by hypertension, hyperglycemia and aging may share the changes with diabetic vascular beds. Endothelin receptor antagonist CPU0213 and ingredients from plant origins such as CPU86017, p-benzyl-tetra-hydro-berberine are effective in attenuating vascular abnormality by normalizing changes of biomarkers in the vascular wall. The early sign of subclinical atherosclerosis presented as an intima media thickness in the carotid may indicate endothelium dysfunction. The reduced ABI (ankle brachial index) has been taken to predict patients at risk for cardiovascular and cerebrovascular events, and an increased risk of mortality from all causes and cardiovascular disease. An application of agents which suppress the activated ET-NADPH oxidase in the vascular wall is beneficial to attenuate vascular abnormalities. It is worth testing the activity of these agents further for the potential in relieving abnormal vascular activity, reducing the risk of morbidity and mortality in patients at risk.

Keywords: diabetes, hypercholesterolemia, ETA, peripheral artery disease, vascular dilatation

\section{Introduction}

Normal vascular activity is essential for maintaining normal function of organs, dependent on a balance of vasoconstrictive and vasodilative substances derived from the vascular endothelium, which mainly include nitric oxide (NO) to dilate and endothelin-1 to constrict the vascular smooth muscle. Endothelium-dependent relaxation directly relates to the biosynthesis and release of NO by the activity of endothelial nitric oxide synthase (eNOS) in the endothelium. Vascular relaxation is encountered by an activated endothelin (ET-1) system, and an over-activated ETA (endothelin receptor A) may contribute to a decline in eNOS activity, resulting in compromised NO bioavailability. ${ }^{1}$

Endothelial dysfunction characterized by impaired endothelium-dependent vasodilatation has been linked to each of the known risk conditions, such as diabetes mellitus, hypertension, dyslipidemia, obesity, cigarette smoking, and aging. The more impaired vascular endothelium, the more severe the risk for cardiovascular events such as coronary infarction; thus, it is likely to take an impaired vascular endothelium as the early stage of coronary infarction. ${ }^{2}$

Regarding the high incidence of morbidity and mortality of cardiovascular disease (CVD), more attention has been paid to the vascular system for getting 
more understanding of the mechanisms in pathologies of complications of diabetes, hyperlipidemia, and stress-related abnormalities.

ET-1 is primarily active as paracrine substance that modulates the basal vascular tone. ETA appears to be at the center in modulating the vascular activity, and an upregulated ETA has been found under multiple conditions: congestive heart failure, hypertension, atherosclerosis, endothelial dysfunction, coronary artery diseases, renal failure, cerebrovascular disease, pulmonary arterial hypertension, and sepsis. ${ }^{3}$ Interestingly, some substances and prescriptions in Traditional Chinese Medicine (TCM) may be effective in reversing endothelial insults by suppressing the activated ETA, such as quercetin, isorhamnetin, or chelerythrine (protein kinase $\mathrm{C}$ (PKC) inhibitor), a modified rehmannia decoction (Liu-wei-di-huang decoction), and total triterpene acids isolated from corni fructus. , $^{1,45}$

CPU86017 (Figure 1A), a derivative from berberine, has been found to be effective in suppressing potassium and calcium channels. ${ }^{6,7}$ It relieves the abnormalities of pulmonary artery hypertension, the abnormalities of calcium modulating proteins in a failing heart, and diabetic cardiomyopathy. ${ }^{8-11}$ There are 2 chiral centers at $7 \mathrm{~N}$ and $13 \mathrm{C} \alpha$ (Figure 1B); consequently, 4 enantiomers that are derived from chiral separation share beneficial effects on the pulmonary hypertension through suppressing the ET receptor. ${ }^{12}$ Besides, a new endothelin receptor antagonist, CPU0213 (Figure 1C), which suppresses both the ETA and ETB, referred as a nonselective (or dual) endothelin receptor antagonist which is effective in suppressing upregulated ET receptors resulting in relieving pulmonary arterial hypertension and vascular abnormalities in diabetic vasculature. ${ }^{1,13}$

\section{Vascular abnormalities and oxidative stress}

Vascular activity depends on a normal endothelium, which releasing vasoactive substances to regulate vascular smooth muscle tone. The ability of formation and release of vascular dilating substance NO in the endothelium is seriously affected by oxidative stress, which appears to reduce the vascular dilatation in response to acetylcholine (ACh) due to less vascular NO released. Hyperglycemia induces advanced glycation end products (AGEs), which have the entity of free radicals affecting the large biological molecules to injure cells. Compromised vascular dilatation by diabetic lesion results from an excess of ROS (reactive oxygen species), which is evidenced that ROS are released from cardiomyocyte while incubated with high glucose. ${ }^{14}$ It has been widely accepted that ROS induced by hyperglycemia contribute to vascular endothelium dysfunction in diabetes. ${ }^{15} \mathrm{~A}$ variety of enzymatic and non-enzymatic sources of ROS exist in the blood vessels, including NADPH oxidase (NOX), mitochondrial electron transport chain, xanthine oxidase, and nitric oxide synthase. Among them the dysfunction of mitochondrial electron transport and activated NOX are critically important in generating oxidative stress causing maladaptive vascular responses. Accumulated evidence indicates that in diabetic rats, upregulated NOX has been found in the thoracic vascular ring of which the vascular relaxation decreases significantly along with an augmented vascular tone developed in response to phenylephrine. ${ }^{16}$ NOX is composed by 2 groups of subunit portions: the catalytic components Nox1, Nox4, gp91 phox; and the modulating components p22phox, p47phox, p67phox, which localize either at the membrane or in the cytosol. ${ }^{17}$ The production of ROS can be augmented while an activation of the enzyme is taking place by moving the cytosolic elements to the membrane, allowing the passage of electron from oxygen atom. Some inducing factors such as ET-1, angiotensin II, and TNF $\alpha$ (tumor necrosis factor $\alpha$ ) are needed for such activation. An increase in ROS in the vascular structure is accompanied with an elevation of inflammatory factors and cytokines, such as leptin and its receptor OBRB (obese receptor type b), and ET- $1 .{ }^{10}$ Indeed, antioxidants reverse these changes, at least in part, of the compromised vascular relaxation. An increase in ROS promotes PKC $\varepsilon$ to be phosphorylated, and $\mathrm{H}_{2} \mathrm{O}_{2}$ is actively implicated in upregulating $\mathrm{PKC} \varepsilon$, and the reaction is substantially
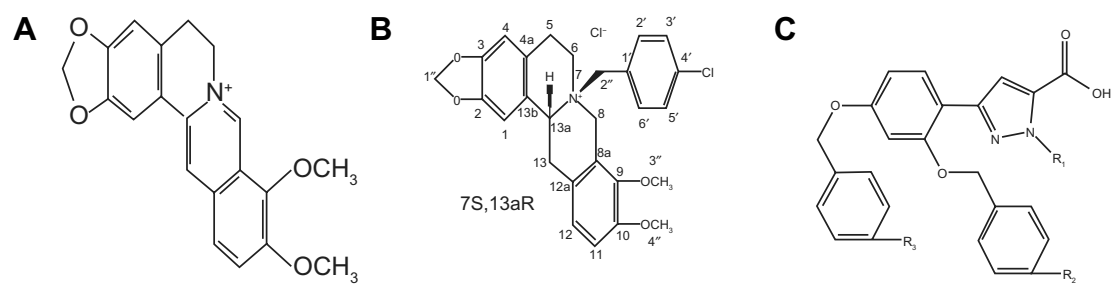

Figure I The chemical structure of berberine (A) CPU86017 (p-benzyl-tetra-hydro-berberine) containing the two chiral centers (B) and CPU0213 a dual endothelin receptor antagonist (C). 
suppressed by either antioxidant tocopherol (vitamin E) or endothelin receptor antagonist CPU0213. ${ }^{18}$

Renal damage relating to microangiopathy is closely linked with hypertension and diabetes and is the consequence of vascular endothelial dysfunction. Evidence has been accumulated to indicate that oxidative stress and inflammation mediate a decrease in the eGFR (estimated glomerular filtration rate) of the kidney, in which an increase in ET-1 is a dominant factor for such prediction. ${ }^{19}$ There is also a link of an early impairment of Alzheimer's disease with an upregulation of regulatory subunit $\mathrm{p} 47 \mathrm{phox}$ and the catalytic subunit gp91phox in vulnerable brain regions, relevant to vascular endothelial dysfunction of the cerebro-artery in the brain. ${ }^{20}$

\section{Diabetic vasculopathy}

Diabetes mellitus causes abnormal vascular activity by presenting insulin resistance and hyperglycemia, which increases AGEs revealed by an increase in HbA1c in the blood stream. AGEs seem to be an entity of free radicals to attack the large molecules by losing their biological activity stemming from the negatively charged. Elevated oxidative stress occurs predominantly in diabetic patients and in animal diabetic models due to hyperglycemia, as well as oxidative modification of atherogenic lipoproteins. ${ }^{20,21}$ The generation of ROS is mainly derived from mitochondrial dysfunction, which increases electron leak from the mitochondrial respiratory chain (MRC), and an activation of NOX results. High levels of glucose and lipids impair the activities of MRC complex enzymes and activate NOX responsible for the generation of ROS. ${ }^{23}$ Increased NOX activity found in diabetic patients contributes to vascular dysfunction. In diabetes, ET-1 levels are elevated and upregulation of ETA and ETB occurs in the vasculature, along with a reduction in NO bioavailability. ${ }^{1,4}$ Activated ET receptors can also be found in diabetic complications such as cardiomyopathy and testopathy. ${ }^{24-26} \mathrm{NOX}$ is stimulated mainly by ETA, with PKC actively involved. ${ }^{27}$ The vasoconstrictive activity of ET-1 is mediated by the activity of NOX, and an activation of the L-type calcium channels by ET-1 causes an influx of calcium ions through activating NOX.$^{28}$ An interaction of ET- 1 with ROS is known as the ET-ROS pathway, which activates the ERK signaling pathway and AP-1 transcriptional factor. ${ }^{29}$ There is a positive feedback mechanism between them via an activation of ERK. ROS activate p38 kinase, which further promotes ROS generation, forming a positive feedback loop to sustain ROS-p38 kinase signaling, which may associate with a reduction in SIRT1 protein expression. Overexpression or activation of SIRT1, an NAD+-dependent protein deacetylase, is sensitive to oxidative signals by drug interventions and significantly reduces the status of oxidative stress. ${ }^{30}$

There are multiple factors implicated in vascular abnormalities under diabetic conditions. ${ }^{31}$ The matrix in the diabetic vascular wall is altered due to changes in MMP2 and MMP9 expression resulting in an increase in accumulated fibroid substances, which may participate in adversely affecting vascular activity. ${ }^{12,16}$ The intercellular gap junction connexin proteins are important for maintaining normal vascular tone through passing small molecules between cells, among which Cx40 (connexin 40) is active in the vascular wall and its downregulation results in a compromised pulmonary vascular dilatation. ${ }^{12}$ Drug intervention normalizes the abnormal $\mathrm{Cx} 43$, leading to a recovery of the cardiovascular abnormalities. ${ }^{22}$ An activated PKC $\varepsilon$ participates in the process of vascular endothelial dysfunction relating to oxidative stress, and hyperphosphorylation of PKCE appears to be involved in insults related to stress or diabetic lesion. ${ }^{32}$

Without sustained hyperglycemia, insulin resistance alone is always found in obese individuals associating with reduced vasodilating response to Ach. On the other hand, while blood glucose has been well controlled with a low HbA1c, however, the morbidity of the cardiovascular system may not be relieved, with mortality remaining high. Diabetic nephropathy as a microvascular complication may progress further. The pathological changes are rarely reversible, but progressive, even the inciting factor has been removed and reduction in functional renal mass leads to hypertrophy of the remaining renal glomeruli and tubules. The adaptations place a heavy burden on the functional renal units leading to glomerular sclerosis and interstitial fibrosis. ${ }^{33}$ Hyperfiltration of the vascular structure of glomeruli is a causal factor to damage the kidney, thus, the end stage of renal disease may occur while blood glucose levels have been well controlled. It indicates that suppression on the cellular insults by diabetic lesions is critical to relieve the progress in diabetic complications. Upregulated ET receptors are related to hyperfiltration and an application of ET receptor blocking agents is effective in reducing the microalbuminuria. ${ }^{34}$ An early application of insulin to keep well controlled blood glucose at the early stage of diabetes is beneficial in attenuating the occurrence of diabetic vascular complications, even hyperglycemia that appears later; the incidence and severity of complications may be reduced due to a mechanism of 'metabolic memory'. 35

Peroxisome proliferator-activated receptor gamma (PPAR $\gamma$ ), a nuclear receptor and transcription factor in the steroid superfamily, is actively implicated in the diabetic vasculature, and PPAR $\gamma$ agonists (the thiazolidinediones) 
are clinically effective in relieving type 2 diabetes. Beyond its activity to increase the sensitivity to insulin, PPAR $\gamma$ plays critical roles in the vasculature, in particular on endothelium-dependent relaxation. ${ }^{36}$ Migraine is associated with a specific vascular risk profile assessed as vascular dysfunction, compared with normal control; in patients with migraine the systolic blood pressure, diastolic blood pressure, blood glucose, and insulin are increased in association with an elevated ET-1 and an increased carotid artery intima thickness, which coincides with a reverse relation to flowmediated vasodilation. ${ }^{37}$ As an indicator for early vascular abnormality in diabetes, the carotid intima-media thickness (IMT) is significantly greater in diabetics without macroangiopathy compared with the normal control, and the increased IMT is significantly progressive over a 30-month follow-up period in diabetics but not in the controls. ${ }^{38}$

\section{Hypertension and stress-induced vascular abnormalities}

Vascular endothelium impaired in primary hypertension shows a reduction in $\mathrm{NO}$ and an increase in ROS contributing to sustained elevated blood pressure. Some antihypertensive agents possess potent blood pressure lowering effect; then, it is interesting to explore if the impaired vascular endothelium in hypertensive patients treated with antihypertensive agents could be back to the normal range. The status of vascular endothelium can be evaluated by an application of Ach and $\mathrm{N}(\mathrm{G})$-monomethyl-L-arginine (L-NMMA) for inducing the stimulated and basal NO release in the endothelium separately. After long-term medication with either valsartan or amlodipine, the amplitude of a lowered blood pressure effect is the same while back to below 140/90 $\mathrm{mmHg}$. The vasodilatory response caused by Ach was significantly increased with valsartan as assessment of the maximal percentage change in the forearm blood flow compared with placebo; however, amlodipine does not produce a significant vasodilatation to Ach. On the other hand, the basal release of NO as tested by L-NMMA is significant in the two medicated groups. It indicates that NO bioavailability and the stimulated NO release from endothelium are varied in response to drug interventions. Both NO dependent and independent pathways can be significantly preserved by valsartan; despite the same antihypertensive activity, amlodipine shows a partial effect on NO bioactivity. ${ }^{39}$ Following one year treatment with either valsartan or amlodipine in a separate clinical test, the baseline blood pressure was similar and the magnitude of the decreases in blood pressure did not differ during the medication between groups. Endothelial function, assessed by flow-mediated vasodilation, was significantly improved with valsartan but not in those treated with amlodipine. The markers for oxidative stress, urinary excretion of 8-isoprostane and 8-hydroxy-2'-deoxyguanosine, were significantly reduced with valsartan against amlodipine..$^{40}$ In another trial hypertensive patients resistant to amlodipine and candesartan were included, and either spironolactone or chlorthalidone was added to improve the antihypertensive activity for 16 weeks. The flow mediated vasodilation test was conducted while both medications caused significant reduction in blood pressure within the normal range and resulted in a decrease in vasodilation seen with chlorthalidone, but not spironolactone. An impaired vasodilative response is likely to be linked with an increase in inflammatory factors. C-reactive protein and uric acid in serum were increased significantly in patients treated with chlorthalidone compared with spironolactone. ${ }^{41}$ Some biomarkers are related to impaired endothelial function in hypertensive patients, a reduction in prostacyclin production, and an increased oxidized LDL associate with a 4.9 higher significant risk of hypertension. ${ }^{42}$ In primary hypertension NOX-derived superoxide, stimulated by AT1 receptors, decreases vascular endothelium released NO due to forming peroxynitrite by reacting with NO. Telmisartan decreases in the aortic levels of the protein expression of gp91, a subunit of NADPH oxidase, resulting in a relief to endothelial dysfunction. ${ }^{43}$ A blockade on the renin-angiotensin-aldosterone system is more beneficial than diuretics in relieving the endothelial dysfunction accounting for an increase in the vasodilating activity.

ETA receptor is activated in the resistance vessels in patients with hypertension. Following an intra-arterial injection of ETA blocker BQ-123 in patients with hypertension, the forearm blood flow is increased significantly, and the vasodilator response caused by BQ-123 in hypertensive subjects, but not in hypercholesterolemic patients or smokers, was significantly greater relative to healthy individuals. Thus, vascular resistance of the peripheral artery is modulated selectively by ETA in hypertension. ${ }^{44}$

Chronic medication of isoproterenol mimicking a stress status related to an increase in sympathetic outflow, causes an impairment of vascular activity which is effectively reversed by endothelin receptor antagonist CPU0213. ${ }^{45}$ The abnormal vascular activity is characterized by an increase in expression of ETA, NOX, and MMP9 in the vasculature which are attenuated by either CPU0213 a blockade on ET receptors and an antioxidant activity by aminoguanidine.

The tone of the coronary arteries is critically dependent on an activation of ET receptors and an increased ET-1 associates with a proportional decrease in NO accompanying coronary endothelial dysfunction. ${ }^{46}$ Vascular abnormalities 
resulting from over-expression of endothelin receptor, leptin, and NOX and stress contribute to an increased risk of CVD (cardiovascular disease).

Endothelium-dependent dilatation of large arteries decreases with aging even in the healthy elderly, and the flowmediated dilatation (FMD) further declines in hypertensive elderly patients, and measurement of carotid artery IMT provides a possible indicator of endothelial dysfunction. In the whole population, maximal Ach-induced vasodilation was inversely related to IMT, and the age-related impairment of endothelium-dependent vasodilation and remodeling of the carotid intima can be attenuated in individuals with regular physical training. ${ }^{47}$ An impairment of vasodilative response of the brachial artery may precede changes in the carotid IMT and left ventricular mass index with aging. ${ }^{48}$

\section{Hyperlipidemia and obesity induced vascular abnormality}

Blood cholesterol lowering drugs reduce the incidence of coronary heart disease, encouraging impetus to global campaigns to search for effective agents in reducing serum cholesterol levels. The plaques found in the arterial walls of patients with hypercholesterolemia indicate an important clue of linking their development with serum cholesterol. The involvement of ET-1 in the process of formation of the plaques in the vasculature has been established in association with a compromised vasodilatation.

Obesity is associated with increased cardiovascular risk due to vascular endothelial dysfunction, mean brachial artery FMD was impaired in obese individuals, and an improvement is significant following sustained weight loss, in association with reducing total and low-density lipoprotein cholesterol, glucose, $\mathrm{HbA} 1 \mathrm{c}$, and C-reactive protein. ${ }^{49}$ It indicates that chronic inflammatory reaction appears to be implicated in the endothelium dysfunction in obese individuals.

More attention has been paid on the early sign of intima damage, as a subclinical atherosclerosis, which accompanies a reduction in the vascular activity. Evidence for existence of early changes relating to atherosclerosis in arterial intima is found as early as in the fetal period. The risk factors of pre-clinical atherosclerosis in childhood are presented as carotid artery IMT by high resolution B-mode ultrasound. The diastolic blood pressure and the pre-clinical atherosclerosis appear to be the risk factors inducing the thickened carotid artery IMT. ${ }^{50}$ The distribution of IMT of the common carotid is not specific to hyperlipidemia, and some hyperlipidemia patients have abnormal IMT of the carotid, whereas in others the femoral artery is more affected. Therefore, in patients with hyperlipidemia, such as familial hypercholesterolemia, a combined assessment of the carotid and femoral arterial walls may be a more accurate way to reveal the early arterial lesion relevant to total atherosclerotic burden. ${ }^{51}$

Primarily, therapeutic agents for the prevention of atherosclerosis are targeted in treating hyperlipidemia and high blood pressure; these are relatively easily measured risk factors. This strategy, however, is of less than optimal efficacy as rates of CVD remain high. Therefore, a biomarker as ET-1 has been intimately implicated in the progression of atherosclerosis. $^{52}$

The compromised vasodilation of the vasculature in patients with hypercholesterolemia can be assessed by measuring flow-dependent vasodilation. ${ }^{53}$ There are many factors resulting in the abnormalities, one of which is likely related to an increase in plasma levels of asymmetric dimethylarginine (ADMA), which is an endogenous inhibitor to NO genesis in vascular endothelium. ${ }^{54}$ An impaired vasodilation assessed by flow-mediated vasodilation in essential hypertension is also related to an increased ADMA in plasma, in association with an elevated $\mathrm{C}$-reactive protein, which indicates an involvement of inflammatory reactions in the vascular wall. ${ }^{55}$ Cerebral vascular activity is damaged by hyperhomocysteinemia and is reversed by overexpression of dimethylarginine dimethylaminohydrolase, which has ADMA-hydrolyzing activity. ${ }^{56}$ The earliest abnormality in the vascular disease represents endothelial dysfunction, which appears to be linked with subsequent atherosclerosis progression as well as in the settings of diabetes mellitus, insulin resistance, dyslipidemia, and hypertension. In elderly subjects, the flow-mediated dilation is conducted by injecting Ach to show positively relating to levels of L-arginine, and L-arginine/ADMA ratio, but not ADMA in plasma in elderly. ${ }^{57}$ Although L-arginine can be converted into vascular NO to dilate vascular smooth muscle, however, an increase in L-arginine levels up to 20 times higher than normal does not affect vasodilative activity and so did with ADMA levels, it may indicate multifaceted factors implicated in the impairment of vascular activity. The subclinical atherosclerosis, high prevalence in airflow limited patients, can be found with carotid IMT and focal atheromatous plaque as indicators of vascular abnormality, which is coincided with increased morbidity and mortality from CVD due to chronic obstructive pulmonary disease..$^{58}$

\section{Peripheral arterial disease detected by ankle brachial index}

The ankle brachial index (ABI), a ratio of blood pressure measured at ankle over the blood pressure in the brachial artery, is 
a known approach for assessing lower-limb peripheral artery disease (PAD). It serves as a marker predicting CVD events. $\mathrm{ABI}$ predicts patients at risk for an increase in mortality from all causes, including CVD: patients with ABI 0.9-1.1, 0.7-0.9, and $<0.7$ had hazard ratios of 1.60, 2.07, and 3.08 for all-cause mortality and 1.89, 2.33, and 4.09 for cardiovascular mortality, respectively. ${ }^{59}$ Abnormal $\mathrm{ABI}$ is associated with an increase in prevalence and recurrence of stroke. ${ }^{60}$ The measurement of ABI amongst patients with ischemic stroke may identify high-risk patients for planning adequate prevention therapies. A modified ABI may be sensitive to find the patients at risk for CVD at the primary medical office. ${ }^{61}$

After acute ischemic stroke (AIS) or transient ischemic attack (TIA), ABI can be used to detect PAD as a marker of generalized atherosclerosis. A high prevalence of AIS, as high as $81.6 \%$, is related to a low ABI and $18.4 \%$ with TIA. ${ }^{62} \mathrm{~A}$ low ABI of $\leq 0.9$ is more likely to be in the elderly or in patients with a history of PAD, hypertension, diabetes, or congestive heart failure. It is also of interest to evaluate mortality across $\mathrm{ABI}$ values and to assess the association with elevated ABI. It has been found that an association between elevated ABI and poor survival is similar to that of low ABI. PAD appears to be an independent risk factor for mortality among patients with elevated $\mathrm{ABI} .{ }^{63} \mathrm{~A}$ low $\mathrm{ABI}$ indicates atherosclerosis and an increased risk of cardiovascular and cerebrovascular events, and aspirin was tested for reducing the risk of cerebrovascular events; however, the outcome was negative. ${ }^{64}$

\section{The ET - NADPH oxidase pathway}

The impaired vasodilative responses and the reduced NO bioavailability may be addressed by an excess of ET-1 resulting in upregulation of ETA in the vascular wall, which is a consequence to diabetes, hypercholesterolemia, hypertension, and aging. A reduction of hyperglycemia, cholesterol-lowering activity, and antihypertensive effect by agents may lead to a relief to the dysfunctional endothelium; however, the protective effect of these procedures may not be completely responded, as revealed in the clinical observations that diabetic nephropathy is relatively refractory to therapy and progressive. ${ }^{65}$

Attention should be paid on the maladaptive changes in the vascular wall in which an activated ET-NOX-PKCE pathway is focused. Angiotensin II(AII), a causal factor to the dysfunction of vascular endothelium, adversely stimulates the activity of the cardiovascular system through producing overt oxidative stress resultant from the activation of NOX. In these effects an activated ET is likely to mediate the activity of the AII through NOX activation as AII-evoked expression of ET-1 in adventitial fibroblasts appears to be mediated, at least in part, by NOX. ${ }^{66}$ The mitochondrial ROS is responsible to mediate the activity of ET-1 in the cardiovascular system. ${ }^{67}$ The vascular activity of AII is closely related to ET-1, coronary vasoconstriction by $\alpha 1$-adrenergic stimulation via the $\alpha$-adrenergic-angiotensin-ET axis which requires NOX mediated signaling in cardiac and vascular cells. ${ }^{68}$ On the cardiac sympathetic nerve endings, ETA and ETB are located, and these two receptors are active in modulating the release of norepinephrine. ${ }^{69}$ The downregulation of FKBP12.6 (FOK binding protein, calstabin 2) as a consequence of isoproterenol or $\mathrm{H}_{2} \mathrm{O}_{2}$ medication is dramatically relieved by an application of endothelin receptor antagonist CPU0213. ${ }^{18}$ Upregulation of NOX by isoproterenol is significant and is separately modulated by ETA and ETB antagonist; however, the activity of ETA is predominant in this regard. ${ }^{70}$ Therefore, it can be recognized that there is a functional link between the activity of sympathetic nervous system, AII and ET-1, and the activity of NOX serves as an important tie in the pathologies relating to an unhealthy vascular system.

In conclusion, agents reducing hyperglycemia and hypercholesterolemia may not be sufficient in relieving the endothelial insults. A reversal of the abnormal endothelial status can be achieved by cell protective effects through suppressing the ET-NOX-PKCE pathway. Agents may relieve the endothelium dysfunction by suppressing this pathway. The progression of the peripheral artery disease and the pathologies implicated in the vascular beds in diabetes, hypercholesterolemia, stress, and aging should be targeted by drug interventions. Therefore, it is likely to reduce the morbidity of CVD and mortality of all causes through normalizing vascular endothelium function. It is hopeful for reducing risk of cerebrovascular and cardiovascular attack by applying ingredients isolated from TCM which suppress the ET-NOX-PKCE axis, and it would be worth doing more tests for the potential to relieve these abnormalities in this regard.

\section{Disclosure}

The authors have no disclosures or conflicts.

\section{References}

1. Su W, Dai DZ, Liu HR, Na T, Dai Y. Upregulated endothelin system in diabetic vascular dysfunction and early retinopathy is reversed by CPU0213 and total triterpene acids from Fructus Corni. Clin Exp Pharmacol Physiol. 2007;34(12):1228-1233.

2. Dobarro D, Gómez-Rubín MC, Sanchez-Recalde A, et al. Current pharmacological approach to restore endothelial dysfunction. Cardiovasc Hematol Agents Med Chem. 2009;7(3):212-222.

3. Shah R. Endothelins in health and disease. Eur J Intern Med. 2007;18(4): 272-282.

4. Romero M, Jiménez R, Sánchez M, et al. Quercetin inhibits vascular superoxide production induced by endothelin-1: role of NADPH oxidase, uncoupled eNOS and PKC. Atherosclerosis. 2009;202(1):58-67. 
5. Liu HR, Tang XY, Dai DZ, Dai Y. Ethanol extracts of Rehmannia complex (Di Huang) containing no Corni fructus improve early diabetic nephropathy by combining suppression on the ET-ROS axis with modulate hypoglycemic effect in rats. J Ethnopharmacol. 2008;118(3): 466-472.

6. Dai DZ. The antiarrhythmic activity of protoberberines in relation to blockade of ion channels. Ion Channel Modulators ID Research Alert. 1997;2:383-390

7. Dai DZ. CPU86017: a novel Class III antiarrhythmic agent with multiple actions at ion channels. Cardiovasc Drug Rev. 2006;24(2):101-115.

8. Zhang TT, Cui B, Dai DZ, Tang XY. Pharmacological efficacy of CPU 86017 on hypoxic pulmonary hypertension in rats: mediated by direct inhibition of calcium channels and antioxidant action, but indirect effects on the ET-1 pathway. J Cardiovasc Pharmacol. 2005;46(6): 727-734.

9. Zhang TT, Cui B, Dai DZ, Su W. CPU 86017, p-chlorobenzyltetrahydroberberine chloride, attenuates monocrotaline-induced pulmonary hypertension by suppressing endothelin pathway. Acta Pharmacol Sin. 2005;26(11):1309-1316.

10. Na T, Huang ZJ, Dai DZ, Zhang Y, Dai Y. Abrupt changes in FKBP12.6 and SERCA2a expression contribute to sudden occurrence of ventricular fibrillation on reperfusion and are prevented by CPU86017. Acta Pharmacol Sin. 2007;28(6):773-782.

11. Qi MY, Feng Y, Dai DZ, Li N, Cheng YS, Dai Y. CPU86017, a berberine derivative, attenuates cardiac failure through normalizing calcium leakage and downregulated phospholamban and exerting antioxidant activity. Acta Pharmacol Sin. 2010;31(2):165-174.

12. Li N, Dai DZ, Dai Y. CPU86017 and its isomers improve hypoxic pulmonary hypertension by attenuating increased ETA receptor expression and extracellular matrix accumulation. Naunyn Schmiedebergs Arch Pharmacol. 2008;378(5):541-552.

13. Cui B, Cheng YS, Dai DZ, Li N, Zhang TT, Dai Y. CPU0213, a nonselective ETA/ETB receptor antagonist, improves pulmonary arteriolar remodeling of monocrotaline-induced pulmonary hypertension in rats. Clin Exp Pharmacol Physiol. 2009;36(2):169-175.

14. Yu T, Sheu SS, Robotham JL, Yoon Y. Mitochondrial fission mediates high glucose-induced cell death through elevated production of reactive oxygen species. Cardiovasc Res. 2008;79(2):341-351.

15. Fatehi-Hassanabad Z, Chan CB, Furman BL. Reactive oxygen species and endothelial function in diabetes. Eur J Pharmacol. 2010;636(1-3):8-17.

16. Xu J, Li N, Dai DZ, Yu F, Dai Y. The endothelin receptor antagonist CPU0213 is more effective than aminoguanidine to attenuate isoproterenol-induced vascular abnormality by suppressing overexpression of NADPH oxidase, ETA, ETB, and MMP9 in the vasculature. J Cardiovasc Pharmacol. 2008;52(1):42-48.

17. Lassegue B, Clempus RE: Vascular NAD(P)H oxidases: specific features, expression, and regulation. Am J Physiol Regul Integr Comp Physiol. 2003;285: R277-R297.

18. Li N, Jia N, Dai DZ, Dai Y. Endothelin receptor antagonist CPU0213 and vitamin E reverse downregulation of FKBP12.6 and SERCA2a: a role of hyperphosphorylation of PKC epsilon. Eur J Pharmacol. 2008; 591(1-3):211-218.

19. Cottone S, Mulè G, Guarneri M, et al. Endothelin-1 and F2-isoprostane relate to and predict renal dysfunction in hypertensive patients. Nephrol Dial Transplant. 2009;24(2):497-503.

20. Bruce-Keller AJ, Gupta S, Parrino TE, et al. NOX activity is increased in mild cognitive impairment. Antioxid Redox Signal. 2010;12(12):1371-1382.

21. Xu M, Dai DZ, Zhang Q, Cheng YS, Dai Y. Upregulated NADPH oxidase contributes to diabetic testicular complication and is relieved by strontium fructose 1,6-diphosphate. Exp Clin Endocrinol Diabetes. 2010;118(7):459-465.

22. Tang XY, Liu Q, Dai DZ, Dai Y. CPU0213, a novel endothelin receptor antagonist, suppresses the upregulation of matrix metalloproteinases and connexin 43 in hyperthyroid myocardium. Pharmacol Rep. 2008; 60(4):524-531.
23. Shen GX. Oxidative stress and diabetic cardiovascular disorders: roles of mitochondria and NADPH oxidase. Can J Physiol Pharmacol. 2010; 88(3):241-248.

24. Qi MY, Xia HJ, Dai DZ, Dai Y. A novel endothelin receptor antagonist CPU0213 improves diabetic cardiac insufficiency attributed to up-regulation of the expression of FKBP12.6, SERCA2a, and PLB in rats. J Cardiovasc Pharmacol. 2006;47(6):729-735.

25. Qi MY, Liu HR, Dai DZ, Li N, Dai Y. Total triterpene acids, active ingredients from Fructus Corni, attenuate diabetic cardiomyopathy by normalizing ET pathway and expression of FKBP12.6 and SERCA2a in streptozotocin-rats. J Pharm Pharmacol. 2008;60(12): $1687-1694$.

26. Zhang Q, Liu HR, Ying HJ, Dai DZ, Tang XY, Dai Y. Strontium fructose 1,6-diphosphate alleviates early diabetic testopathy by suppressing abnormal testicular matrix metalloproteinase system in streptozocin-treated rats. J Pharm Pharmacol. 2009;61(2): 229-236.

27. Matsuo J, Oku H, Kanbara Y, Kobayashi T, Sugiyama T, Ikeda T. Involvement of NADPH oxidase and protein kinase $\mathrm{C}$ in endothelin-1induced superoxide production in retinal microvessels. Exp Eye Res. 2009;89(5):693-699.

28. Zeng Q, Zhou Q, Yao F, O'Rourke ST, Sun C. Endothelin-1 regulates cardiac L-type calcium channels via $\mathrm{NAD}(\mathrm{P}) \mathrm{H}$ oxidase-derived superoxide. J Pharmacol Exp Ther. 2008;326(3):732-738.

29. Duan J, Xu H, Dai S, et al. Phytoestrogen alpha-zearalanol inhibits homocysteine-induced endothelin-1 expression and oxidative stress in human umbilical vein endothelial cells. Atherosclerosis. 2008;197(2): $549-555$.

30. Hong EH, Lee SJ, Kim JS, et al. Ionizing radiation induces cellular senescence of articular chondrocytes via negative regulation of SIRT1 by p38 kinase. J Biol Chem. 2010;285(2):1283-1295.

31. Deedwania P, Srikanth S. Diabetes and vascular disease. Expert Rev Cardiovasc Ther. 2008;6(1):127-138.

32. Potenza MA, Gagliardi S, Nacci C, Carratu' MR, Montagnani M. Endothelial dysfunction in diabetes: from mechanisms to therapeutic targets. Curr Med Chem. 2009;16(1):94-112.

33. Chronic renal disease, Chapter 3, Disease of kidney and Urinary system, In: Tierney LM, McPhee SJ, Papadakis MA, editors. Current Medical Diagnosis and Treatment. McGraw Hill/The People Health Publication, Beijing, China; 2002:283-290.

34. Neuhofer W, Pittrow D. Role of endothelin and endothelin receptor antagonists in renal disease. Eur J Clin Invest. 2006;36:78-88.

35. Drzewoski J, Kasznicki J, Trojanowski Z. The role of "metabolic memory" in the natural history of diabetes mellitus. Pol Arch Med Wewn. 2009;119(7-8):493-500.

36. Matsumoto T, Kobayashi T, Kamata K. Relationships among ET-1, PPARgamma, oxidative stress and endothelial dysfunction in diabetic animals. J Smooth Muscle Res. 2008;44(2):41-55.

37. Hamed SA, Hamed EA, Ezz Eldin AM, Mahmoud NM. Vascular risk factors, endothelial function, and carotid thickness in patients with migraine: relationship to atherosclerosis. $J$ Stroke Cerebrovasc Dis. 2010;19(2):92-103.

38. Kalogeropoulou K, Mortzos G, Migdalis I, et al. Carotid atherosclerosis in type 2 diabetes mellitus: potential role of endothelin-1, lipoperoxides, and prostacyclin. Angiology. 2002;53(3):279-285.

39. Tzemos N, Lim PO, MacDonald TM. Valsartan improves endothelial dysfunction in hypertension: a randomized, double-blind study. Cardiovasc Ther. 2009;27(3):151-158.

40. Hirooka Y, Kimura Y, Sagara Y, Ito K, Sunagawa K. Effects of valsartan or amlodipine on endothelial function and oxidative stress after one year follow-up in patients with essential hypertension. Clin Exp Hypertens. 2008;30(3):267-276.

41. Yamanari H, Nakamura K, Miura D, Yamanari S, Ohe T. Spironolactone and chlorthalidone in uncontrolled elderly hypertensive patients treated with calcium antagonists and angiotensin II receptor-blocker: effects on endothelial function, inflammation, and oxidative stress. Clin Exp Hypertens. 2009;31(7):585-594. 
42. Kuklinska AM, Mroczko B, Musial WJ, et al. Diagnostic biomarkers of essential arterial hypertension: the value of prostacyclin, nitric oxide, oxidized-LDL, and peroxide measurements. Int Heart J. 2009;50(3):341-351.

43. Kagota S, Tada Y, Kubota Y, et al. Peroxynitrite is involved in the dysfunction of vasorelaxation in SHR/NDmcr-cp rats, spontaneously hypertensive obese rats. J Cardiovasc Pharmacol. 2007;50(6):677-685.

44. Nohria A, Garrett L, Johnson W, Kinlay S, Ganz P, Creager MA. Endothelin-1 and vascular tone in subjects with atherogenic risk factors. Hypertension. 2003;42(1):43-48.

45. Luo L, Dai DZ, Dai Y. Effect of the endothelin receptor antagonist CPU0213, and its modulation by rifampin, on cardiac and vascular tissue following chronic isoproterenol treatment. Clin Exp Pharmacol Physiol. 2008;35(7):757-765.

46. Nguyen A, Thorin-Trescases N, Thorin E. Working under pressure: coronary arteries and the endothelin system. Am J Physiol Regul Integr Comp Physiol. 2010 Mar 17. [Epub ahead of print]

47. Galetta F, Franzoni F, Virdis A, et al. Endothelium-dependent vasodilation and carotid artery wall remodeling in athletes and sedentary subjects. Atherosclerosis. 2006;186(1):184-192.

48. Djuric D, Popovic Z, Petrovic J, Bojic M. Age-related progressive brachial artery endothelial dysfunction precedes the changed carotid and left ventricular geometry in healthy humans. Angiology. 1999;50(7):555-561.

49. Bigornia SJ, Mott MM, Hess DT, et al. Long-term successful weight loss improves vascular endothelial function in severely obese individuals. Obesity (Silver Spring). 2010;18(4):754-759.

50. Yang XZ, Liu Y, Mi J, Tang CS, DU JB. Pre-clinical atherosclerosis evaluated by carotid artery intima-media thickness and the risk factors in children. Chin Med J (Engl). 2007;120(5):359-362.

51. Wittekoek ME, de Groot E, Prins MH, Trip MD, Büller HR, Kastelein JJ. Differences in intima-media thickness in the carotid and femoral arteries in familial hypercholesterolemic heterozygotes with and without clinical manifestations of cardiovascular disease. Atherosclerosis. 1999;146(2):271-279.

52. Little PJ, Ivey ME, Osman N. Endothelin-1 actions on vascular smooth muscle cell functions as a target for the prevention of atherosclerosis. Curr Vasc Pharmacol. 2008;6(3):195-203.

53. Vladimirova-Kitova LG, Manukov IH, Sirakova I, Deneva TI. Evaluation of the relationship between flow-mediated vasodilation and some atherogenic risk markers in severe hypercholesterolemia. Folia Med (Plovdiv). 2008;50(1):22-28.

54. Xia W, Feng W, Guan M, Yu Y, Li J, Qu X. Increased levels of asymmetric dimethylarginine and C-reactive protein are associated with impaired vascular reactivity in essential hypertension. Clin Exp Hypertens. 2010;32(1):43-48.

56. Rodionov RN, Dayoub H, Lynch CM, et al. Overexpression of dimethylarginine dimethylaminohydrolase protects against cerebral vascular effects of hyperhomocysteinemia. Circ Res. 2010;106(3): 551-558.

56. Lind L, Larsson A, Teerlink T. L-Arginine is related to endotheliumdependent vasodilation in resistance and conduit arteries in divergent ways. The Prospective Investigation of the Vasculature in Uppsala Seniors (PIVUS) study. Atherosclerosis. 2009;203(2): 544-549.
57. Gates PE, Boucher ML, Silver AE, Monahan KD, Seals DR. Impaired flow-mediated dilation with age is not explained by L-arginine bioavailability or endothelial asymmetric dimethylarginine protein expression. J Appl Physiol. 2007;102(1):63-71.

58. Iwamoto H, Yokoyama A, Kitahara Y, et al. Airflow limitation in smokers is associated with subclinical atherosclerosis. Am J Respir Crit Care Med. 2009;179(1):35-40.

59. Zheng L, Li J, Hu D, et al. Association of low ankle-brachial index with mortality in patients with ischemic heart disease. J Atheroscler Thromb. 2010 Mar 13. [Epub ahead of print]

60. Purroy F, Coll B, Oró M, et al. Predictive value of ankle brachial index in patients with acute ischaemic stroke. Eur J Neurol. 2009 Nov 24. [Epub ahead of print]

61. Topakian R, Nanz S, Rohrbacher B, Koppensteiner R, Aichner FT; for the OECROSS Study Group. High prevalence of peripheral arterial disease in patients with acute ischaemic stroke. Cerebrovasc Dis. 2010;29(3):248-254.

62. Oksala NK, Viljamaa J, Saimanen E, Venermo M; for the ATTAC study group. Modified ankle-brachial index detects more patients at risk in a Finnish primary health care. Eur J Vasc Endovasc Surg. 2010;39(2):227-233.

63. Suominen V, Uurto I, Saarinen J, Venermo M, Salenius J. PAD as a risk factor for mortality among patients with elevated ABI - a clinical study. Eur J Vasc Endovasc Surg. 2010;39(3):316-322.

64. Fowkes FG, Price JF, Stewart MC, et al. Aspirin for prevention of cardiovascular events in a general population screened for a low ankle brachial index: a randomized controlled trial. JAMA. 2010;303(9):841-848.

65. Al-Wakeel JS, Hammad D, Al Suwaida A, Mitwalli AH, Memon NA, Sulimani F. Microvascular and macrovascular complications in diabetic nephropathy patients referred to nephrology clinic. Saudi J Kidney Dis Transpl. 2009;20(1):77-85.

66. An SJ, Boyd R, Zhu M, Chapman A, Pimentel DR, Wang HD. NADPH oxidase mediates angiotensin II-induced endothelin-1 expression in vascular adventitial fibroblasts. Cardiovasc Res. 2007;75(4):702-709.

67. De Giusti VC, Correa MV, Villa-Abrille MC, et al. The positive inotropic effect of endothelin-1 is mediated by mitochondrial reactive oxygen species. Life Sci. 2008;83(7-8):264-271.

68. Yamaguchi O, Kaneshiro T, Saitoh S, Ishibashi T, Maruyama Y, Takeishi Y. Regulation of coronary vascular tone via redox modulation in the alpha1-adrenergic-angiotensin-endothelin axis of the myocardium. Am J Physiol Heart Circ Physiol. 2009;296(1):H226-H232.

69. Isaka M, Kudo A, Imamura M, Kawakami H, Yasuda K. Endothelin receptors, localized in sympathetic nerve terminals of the heart, modulate norepinephrine release and reperfusion arrhythmias. Basic Res Cardiol. 2007;102(2):154-162.

70. Peng HJ, Dai DZ, Ji H, Dai Y. The separate roles of endothelin receptors participate in remodeling of matrix metalloproteinase and connexin 43 of cardiac fibroblasts in maladaptive response to isoproterenol. Eur $J$ Pharmacol. 2010;634(1-3):101-106.
Vascular Health and Risk Management

\section{Publish your work in this journal}

Vascular Health and Risk Management is an international, peerreviewed journal of therapeutics and risk management, focusing on concise rapid reporting of clinical studies on the processes involved in the maintenance of vascular health; the monitoring, prevention and treatment of vascular disease and its sequelae; and the involvement of

\section{Dovepress}

metabolic disorders, particularly diabetes. This journal is indexed on PubMed Central and MedLine. The manuscript management system is completely online and includes a very quick and fair peer-review system, which is all easy to use. Visit http://www.dovepress.com/ testimonials.php to read real quotes from published authors. 